\title{
Escala de satisfacción con la vida (SWLS): análisis de validez, confiabilidad y baremos para estudiantes universitarios de Bogotá
}

\author{
Satisfaction with life Scale (SWLS): validity, reliability and \\ assessment analysis in college students from Bogotá (Col) as \\ sample
}

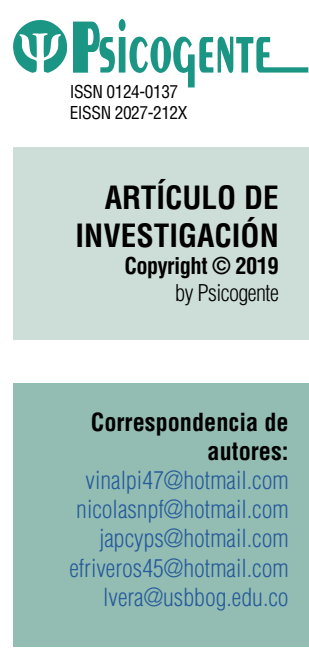

Recibido: $16-03-18$ Aceptado: $31-01-19$ Publicado: 01-07-19

Cómo citar este artículo (APA):

Vinaccia Alpi, E., Parada, N., Quiceno, J.M., Riveros Munévar, F., \& Vera Maldonado, L.A. (2019). Escala de satisfacción con la vida (SWLS): análisis de

validez, confiabilidad y baremos para estudiantes universitarios de Bogotá. Psicogente 22(42), 1-20. https://doi.org/10.17081/psico.22.42.3468

\author{
Stefano Vinaccia Alpi (iD) - Nicolás Parada (iD \\ Fundación Universitaria Sanitas, Bogotá, Colombia \\ Japcy Margarita Quiceno iD \\ Universidad de Medellín, Medellín, Colombia \\ Fernando Riveros Munévar (iD \\ Universidad de La Sabana, Chía, Colombia \\ Luis Andersen Vera Maldonado iD \\ Universidad de San Buenaventura, Bogotá, Colombia
}

Resumen

Objetivo: Analizar la validez, confiabilidad y baremos de la escala de satisfacción con la vida (SWLS) por Vázquez, Duque y Hervás (2013) en estudiantes universitarios de la ciudad de Bogotá.

Método: Participaron en el estudio 121 universitarios de ambos sexos entre los 18 y 45 años. Se utilizó como instrumento para esta investigación la versión traducida y adaptada al español del SWLS por Vázquez, Duque y Hervás (2013). El estudio fue de corte transversal, de tipo instrumental y con un método psicométrico.

Resultados: El análisis factorial denota un solo factor que explica el 62,3 \% de la varianza total acumulada, modelo que fue confirmado con ecuaciones estructurales y que concuerda con la propuesta teórica de la escala, así como un alfa de Cronbach general de 0,839, correlaciones positivas entre todos los reactivos y apropiada concordancia entre los elementos y el puntaje total corregido.

Conclusiones: La Escala de satisfacción con la vida (SWLS) muestra una alta confiabilidad, apropiada consistencia interna entre los reactivos, correlaciones entre elementos y el puntaje total corregido, y concordancia en la estructura factorial con la versión original, lo que indica una adecuada validez de constructo, con apropiada confiabilidad y consistencia en la muestra de estudiantes universitarios, recomendando su aplicación en esta muestra.

Palabras clave: validez de las pruebas, reproducibilidad de resultados, escala de satisfacción con la vida (SWLS), psicometría.

Abstract

Objective: Analyze the validity, reliability and assessment of the Satisfaction with life Scale (SWLS) Duque and Hervás (2013) in college students from Bogotá as sample.

Method: 121 college students both males and females between 18 and 45 years old were sampled in this study. For this research, the translated and adapted version of SWLS by Vázquez, Duque and Hervás (2013) were used as instruments. A cross-sectional and instrumental type and a psychometric method were conducted for this research.

Results: The factorial analysis shows a single factor that explains $62,3 \%$ of the total cumulative variance; this model was confirmed by using structural equations and which are consistent with the theoretical 
proposal of the scale, and also a general Cronbach's alpha $=0,839$, positive correlations among all reagents and appropriate matching between elements and the correct overall score.

Conclusions: The satisfaction with life scale (SWLS) shows a high reliability, appropriated internal consistency among the items, concordance between reagents, correlation between elements and the correct overall score, and concordance between the factorial structure with the original version, showing an adequate construct validity, appropriate reliability and consistency in college students sampling, recommending its application.

Key words: test validity, reproducibility of results, satisfaction with life scale (SWLS), psychometric method.

\section{INTRODUCCIÓN}

El modelo de Bienestar Subjetivo es un constructo teórico desarrollado en los años 80 del pasado siglo por el psicólogo norteamericano Ed Diener que describe cómo es el proceso de las personas para alcanzar y experimentar bienestar. El Bienestar Subjetivo según su autor está formado con diferentes componentes: la satisfacción por su propia vida (juicio global), satisfacción por los diferentes ámbitos (por ejemplo, por el trabajo), las emociones positivas y un nivel bajo de emociones negativas (Diener, 2000). Al respecto Diener $(1984,1994)$ identifica tres grandes aspectos que caracterizan el bienestar: en primer lugar es subjetivo, en segundo lugar no es la mera ausencia de factores negativos y finalmente incluye una evaluación global y no momentánea de la propia vida. Es importante recordar aquí el juicio de cuánto una persona está satisfecha de su propia vida basado en la comparación entre el propio estado y un estándar de comparación que todo individuo define personalmente según sus ideales (Diener, 1984). El aspecto afectivo del bienestar hedónico consiste entonces en estados de ánimo y emociones, tanto positivas como negativas, mientras el componente cognitivo del bienestar hedónico, a menudo denominado satisfacción con la vida, es un proceso crítico en el que los individuos evalúan la calidad de sus vidas en función de su propio conjunto de criterios (Pavot \& Diener, 1993).

La satisfacción con la vida puede entonces ser definida como un juicio global cognitivo de la propia vida. En este sentido hay que enfatizar que la evaluación realizada no es una evaluación basada en parámetros objetivos, sino que más bien depende de la comparación de las circunstancias vitales, con los propios patrones internos (Diener, 1984; Diener, Emmons, Larsen, \& Griffin, 1985; Pavot \& Diener, 1993; Diener, 2000). La satisfacción con la vida, es la percepción personal de bienestar o felicidad; en otras palabras, es la valoración de la vida propia, en relación con los propios objetivos, expectativas o intereses mediados directamente por el contexto cultural en el que se habite (Diener, Wolsic, \& Fujita, 1995). 
La escala de satisfacción con la vida (SWLS) es probablemente la medida más utilizada de satisfacción con la vida en todo el mundo (Diener et al., 1985; Pavot \& Diener, 1993). La escala consta de cinco ítems y utiliza un formato de respuesta tipo Likert. El análisis factorial exploratorio sugiere que la escala es unidimensional. Al respecto, uno de los primeros análisis elaborado por Diener et al. (1985) encontró un solo factor que representa el $66 \%$ de la varianza, y resultados similares han sido reportados por Pavot \& Diener (1993). Un factor de estructura única también en las numerosas validaciones que se han desarrollado en todo el mundo en casi todos los idiomas y dialectos de diferentes grupos étnicos tanto en población juvenil, como adulta y de la tercera edad con y sin patologías/limitaciones específicas (Pavot \& Diener, 1993; Diener, \& Eunkook, 2003; Diener, Tay \& Oishi, 2013; Kowalska, Gorackzo, Javorwka \& Szczepańska, 2017; Cwiek et al., 2017; Tsaousides et al., 2017; Yoder, Mcfall \& Glaser, 2017; Sarid et al 2017; Tessier, Blanchin \& Sibille, 2017; Lovereide \& Hagell, 2016; Mehamedagic, Santen, Jorgensen \& Acosta, 2016).

En el mundo en los últimos años se han desarrollado numerosas validaciones, análisis psicométricos y estudios con esta escala; véase entre otras Polonia (Buliński \& Blachnio, 2017; Jalali, et al., 2016), España (Vázquez, Duque \& Hervás, 2013; Díaz, Stavraski, Blanco \& Gandarillas, 2015), Estados Unidos (Goverman et al., 2016), México (Padros, Gutiérrez \& Medina, 2015; López-Ortega, Torres-Castro \& Rosas-Carrasco, 2016), Chile (Vera-Villarroel, Urzúa, Pavez, Celis-Atenas \& Silva, 2012), Brasil (Gouveia, Milfont, Nunes \& Peçanha de Miranda, 2009; Paiva et al., 2016), Argentina (Moyano, Martínez \& Muñoz, 2013). En Colombia a pesar de que se ha utilizado ampliamente la escala, véase Gómez, Villegas, Barrera y Cruz (2007) y Salazar (2009) en poblaciones universitarias; Nader, Peñate y Sánchez (2014) con trabajadores de empresas, y Díaz et al., (2015) con población general; Arango-Lasprila et al. (2010); Arango-Lasprilla, Moreno, Rogers y Francis (2009); Sutter et al. (2014) con cuidadores de personas con enfermedades, y Murillo \& Molero (2016) con migrantes colombianos en los Estados Unidos, no se conocen estudios en los que se analicen sus propiedades psicométricas. Por lo tanto, el objetivo de esta investigación fue determinar la validez, confiabilidad y establecer los baremos de la escala de Satisfacción con la vida (SWLS) en estudiantes universitarios de la ciudad de Bogotá, Colombia.

\section{MÉTODO}

\subsection{Tipo de estudio}

El presente es un estudio de corte transversal y de tipo instrumental (Montero \& León, 2005). 


\subsection{Participantes}

Se contó con la participación de 121 adultos jóvenes, siendo 37 hombres y 84 mujeres, pertenecientes a universidades privadas y públicas de la ciudad de Bogotá, con edades comprendidas entre los 18 y los 45 años (media=20,73; d.t.=3,823), obtenidos por medio de un muestreo no probabilístico por sujetos disponibles. El tamaño de la muestra es suficiente para realizar los análisis estadísticos, tanto por el poder del efecto como los análisis factoriales posteriormente mostrados. Más información de la muestra se encuentra en la tabla 1.

Tabla 1.

Datos sociodemográficos de la muestra.

\begin{tabular}{|c|c|c|}
\hline VARIABLE & $\mathbf{N}$ & $\%$ \\
\hline \multicolumn{3}{|l|}{ Sexo } \\
\hline Hombre & 37 & 30,6 \\
\hline Mujer & 84 & 69,4 \\
\hline \multicolumn{3}{|l|}{ Estado Civil } \\
\hline Soltero & 118 & 97,5 \\
\hline Casado & 3 & 2,5 \\
\hline \multicolumn{3}{|l|}{ Trabajo } \\
\hline Empleado & 24 & 19,8 \\
\hline Independiente & 97 & 80,2 \\
\hline \multicolumn{3}{|l|}{ Vive } \\
\hline Solo & 10 & 8,3 \\
\hline Familiares & 97 & 88,4 \\
\hline Amigos & 14 & 11,6 \\
\hline \multicolumn{3}{|l|}{ Estrato } \\
\hline Uno & 2 & 1,7 \\
\hline Dos & 25 & 20,7 \\
\hline Tres & 59 & 48,8 \\
\hline Cuatro & 30 & 24,8 \\
\hline Cinco & 4 & 3,3 \\
\hline Seis & 1 & 0,8 \\
\hline \multicolumn{3}{|l|}{ Edad } \\
\hline $18-25$ & 110 & 90,9 \\
\hline $26-35$ & 9 & 7,4 \\
\hline $36-45$ & 2 & 1,7 \\
\hline
\end{tabular}

\subsection{Instrumento.}

La Escala de Satisfacción con la Vida (SWLS) de Diener et al. (1985) es un instrumento breve de cinco elementos, en escala Likert de valoración de 7 
puntos, desde 1 como "muy en desacuerdo" y 7 como "muy de acuerdo", con puntuaciones entre 5 y 35 , y que evalúa la satisfacción general que tiene el individuo con su vida, entendiendo que mayor puntuación refleja mayor satisfacción.

Para esta investigación se utilizó la versión traducida y adaptada al español por Vázquez, Duque y Hervás (2013) y los datos normativos propuestos por Pavot \& Diener (1993), quienes clasifican así: 31-35, muy satisfecho; 26-30, satisfecho; $21-25$, un poco satisfecho, 20 , neutral, $15-19$, un poco insatisfecho, 10-14, insatisfecho, y 5-9, muy insatisfecho.

\subsection{Procedimiento}

Antes de iniciar la fase de campo de la investigación que implicaba la aplicación de la escala y posterior a la firma de los consentimientos informados, se llevó a cabo la evaluación cultural del instrumento según los criterios de Alexandre \& Guirardello (2002). El trabajo de adaptación cultural consistió en la aplicación de la escala en un grupo de 10 adultos jóvenes de ambos sexos. Luego de responder los cuestionarios fueron entrevistados para identificar palabras o preguntas de difícil comprensión, evaluar la aceptabilidad y hacer comentarios de una forma general, registrándose además el tiempo necesario para completarlo y la comprensión respectiva en el sistema de respuesta de cada ítem de los cuestionarios. No se presentaron dificultades que ameritaran cambios; los participantes reportaron entendimiento y comprensión de los ítems. Posterior a ello se procedió a realizar las aplicaciones de la escala en la muestra total.

Después de obtener las autorizaciones de tres instituciones universitarias de la ciudad de Bogotá y los docentes de los grupos, se procedió a obtener los consentimientos informados de los participantes, seguido de las aplicaciones autodiligenciadas del Cuestionario de datos personales, como de instrumento SWLS. El promedio de cada aplicación fue de cinco minutos y se realizaba de forma individual o grupal (cuando se hicieron aplicaciones grupales, se verificaba que no compartieran información entre los participantes, para garantizar la validez de los datos).

\subsection{Análisis de datos}

Los datos obtenidos se analizaron por medio del paquete estadístico SPSS versión 23 para los análisis factoriales exploratorios y de confiabilidad, y con el aplicativo Amos para el análisis factorial confirmatorio. 


\subsection{Conflicto de interés}

No hay conflicto de intereses.

\section{RESULTADOS}

Con el fin de establecer si el tamaño muestral es suficiente para realizar el análisis factorial, se encontró un KMO de 0,847 y una significancia de la prueba de esfericidad de Bartlett $p<0,001$, estadísticos que soportan el tamaño muestral y que denotan una apropiada matriz de correlaciones para llevar a cabo análisis factoriales, por lo que se realiza dicho análisis factorial por medio de la extracción de componentes principales. En primera medida las comunalidades de cada reactivo, para verificar si es apropiado conservarlos en su totalidad, oscilaron entre 0,549 a 0,744 , lo que permite indicar que todos los reactivos dentro de la escala tienen una extracción suficiente (mayor a 0,3) para que todos permanezcan, procediendo entonces a determinar el número de factores que componen la escala para la muestra aplicada, para lo cual se encontró un único factor que explica el 62,3 \% de la varianza total acumulada.

Con el fin de realizar el análisis factorial confirmatorio de la estructura obtenida, se procedió a realizar el análisis a través del aplicativo Amos, con ecuaciones estructurales. La figura 1 muestra el modelo gráfico confirmado, con sus cargas estandarizadas, mientras que la Tabla 2 nos permite ver los coeficientes obtenidos para el modelo confirmado.

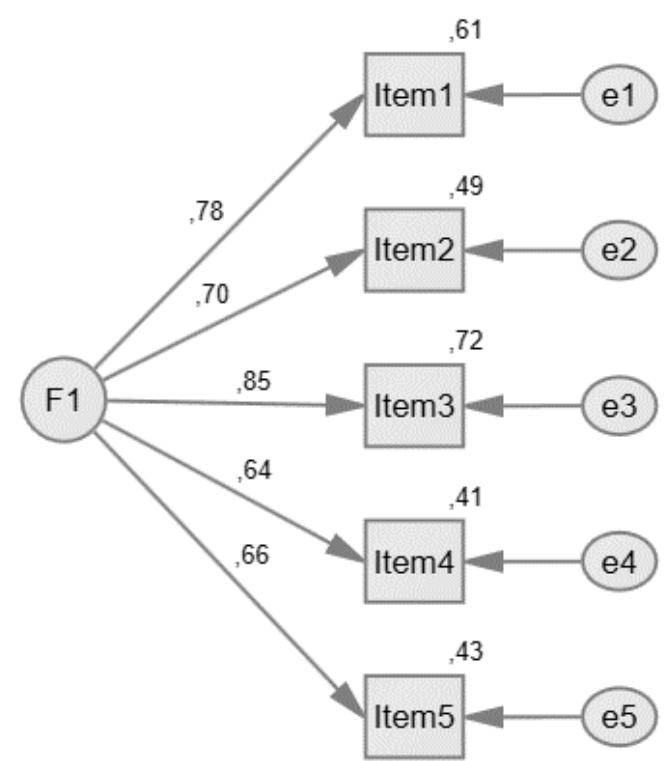

Figura 1. Modelo factorial confirmatorio, con cargas estandarizadas. 
Tabla 2.

Coeficientes obtenidos para el análisis factorial confirmatorios

\begin{tabular}{ccccccc}
\hline $\mathbf{X}^{2}$ & G.L. & $\mathbf{X}^{2} /$ G.L. & GFI & AGFI & CFI & RMSEA \\
\hline 4,625 & 5 & 0,925 & 0,988 & 0,965 & 1 & 0,000
\end{tabular}

Como puede observarse en la figura 1 , se hallaron varianzas positivas y adecuadas (superiores a 0,3 ) de cada reactivo a la carga unifactorial, afirmando la viabilidad del modelo, mientras que la Tabla 2 refiere coeficientes que verifican la unidimensionalidad de la escala (X²/G.L. $<3 ; \mathrm{GFI}>0,95$; AGF>0,95; CFI>0,95; RMSEA<0,05). Adicional, y con el fin de determinar las covariaciones entre los reactivos, se muestran en la Tabla 3 dichas covariaciones entre reactivos.

Tabla 3.

Covariaciones entre ítems.

\begin{tabular}{|c|c|c|c|c|c|}
\hline \multirow{2}{*}{ ÍTEM } & \multirow{2}{*}{ COEFICIENTE } & \multicolumn{4}{|c|}{ ÍTEMS } \\
\hline & & 2 & 3 & 4 & 5 \\
\hline \multirow{2}{*}{1} & r & 0,541 & 0,658 & 0,518 & 0,517 \\
\hline & Sig & 0,000 & 0,000 & 0,000 & 0,000 \\
\hline \multirow{2}{*}{2} & $r$ & - & 0,620 & 0,457 & 0,402 \\
\hline & Sig & - & 0,000 & 0,000 & 0,000 \\
\hline \multirow{2}{*}{3} & r & - & - & 0,515 & 0,569 \\
\hline & Sig & - & - & 0,000 & 0,000 \\
\hline \multirow{2}{*}{4} & $r$ & - & - & - & 0,465 \\
\hline & Sig & - & - & - & 0,000 \\
\hline
\end{tabular}

En la Tabla 3, se encontraron correlaciones positivas y superiores a 0,3 entre todos los reactivos de la escala, lo que muestra la consistencia de la misma. Por otro lado, la Tabla 4 deja ver la tendencia de respuesta de los participantes en cada reactivo y opción de respuesta, así como la media, desviación, alfa de Cronbach, asimetría y curtosis de ellos, mostrando que todas las opciones de respuesta fueron utilizadas por los participantes, y que hay apropiados datos de confiabilidad de la escala. Cabe resaltar que se encontró un alfa de Cronbach general de 0,839 .

Tabla 4.

Datos de respuesta y de confiabilidad de la escala.

\begin{tabular}{|c|c|c|c|c|c|c|c|c|c|c|c|c|}
\hline \multirow{2}{*}{ ÍTEMS } & \multicolumn{7}{|c|}{ \% DE OPCIONES DE RESPUESTA } & \multirow{2}{*}{ MEDIA } & \multirow{2}{*}{ DT } & \multirow{2}{*}{ ALFA } & \multirow{2}{*}{ ASIMETRÍA } & \multirow{2}{*}{ CURTOSIS } \\
\hline & 1 & 2 & 3 & 4 & 5 & 6 & 7 & & & & & \\
\hline 1 & 0,8 & 6,6 & 5 & 8,3 & 19 & 45,5 & 14,9 & 5,34 & 1,4 & 0,80 & $-1,18$ & 0,82 \\
\hline 2 & 0,8 & 1,7 & 1,7 & 11,6 & 12,4 & 51,2 & 20,7 & 5,69 & 1,15 & 0,82 & $-1,43$ & 2,64 \\
\hline 3 & 0,8 & 3,3 & 2,5 & 9,9 & 15,7 & 31,4 & 36,4 & 5,76 & 1,35 & 0,79 & $-1,27$ & 1,39 \\
\hline 4 & 0,0 & 6,6 & 1,7 & 12,4 & 19 & 33,1 & 27,3 & 5,52 & 1,4 & 0,82 & $-1,01$ & 0,49 \\
\hline 5 & 4,1 & 9,9 & 7,4 & 9,1 & 19,8 & 28,9 & 20,7 & 5 & 1,76 & 0,83 & $-0,77$ & $-0,46$ \\
\hline Total & - & - & - & - & - & - & - & 27,31 & 5,59 & 0,84 & $-0,92$ & 0,49 \\
\hline
\end{tabular}


Adicional, se muestra en la Tabla 5 la tendencia de respuesta de los estudiantes universitarios que participaron en la investigación, donde se encuentra que ellos tienden a una respuesta positiva sobre la satisfacción con su vida, en tanto que el $69,4 \%$ de la muestra refiere una valoración de satisfecho o muy satisfecho.

Tabla 5.

Tendencia de respuesta en los participantes, según opción de respuesta.

\begin{tabular}{ccc}
\hline NIVEL & N & $\%$ \\
\hline Muy Insatisfecho & 1 & 0,8 \\
Insatisfecho & 1 & 0,8 \\
Ligeramente Insatisfecho & 9 & 7,4 \\
Neutro & 5 & 4,1 \\
Ligeramente satisfecho & 21 & 17,4 \\
Satisfecho & 49 & 40,5 \\
Muy Satisfecho & 35 & 28,9 \\
\hline
\end{tabular}

Finalmente se procedió a revisar los datos normativos para la muestra, por lo que inicialmente se determinó si existían diferencias entre las puntuaciones según el sexo, por lo que se usó el estadístico no paramétrico $U$ de Mann-Whitney. Los resultados se muestran en la Tabla 6.

Tabla 6.

Análisis de las diferencias en las puntuaciones

\begin{tabular}{ccccccc}
\hline & ÍTEM1 & ÍTEM2 & ÍTEM3 & ÍTEM4 & ÍTEM5 & TOTAL \\
\hline U de Mann-Whitney & 1470,500 & 1493,000 & 1411,500 & 1287,000 & 1550,500 & 1334,000 \\
Sig. Asintót. (bilateral) & 0,801 & 0,710 & 0,402 & 0,120 & 0,984 & 0,307 \\
\hline a. Variable de agrupación: sexo & & & & \\
\hline
\end{tabular}

Dado que, según los análisis estadísticos de la Tabla 6, no existen diferencias entre las puntuaciones de hombres y mujeres, en la Tabla 7 se adjuntan las tablas normativas para calificar los puntajes de los jóvenes colombianos.

Tabla 7.

Percentiles para las puntuaciones directas obtenidas en la escala.

\begin{tabular}{cccc}
\hline PUNTUACIÓN DIRECTA & PERCENTIL & PUNTUACIÓN DIRECTA & PERCENTIL \\
\hline $9-11$ & 1 & 29 & 55 \\
$12-15$ & 2 & 29 & 60 \\
16 & 3 & 30 & 65 \\
16 & 4 & 31 & 70 \\
17 & 5 & 31 & 75 \\
$18-19$ & 10 & 32 & 80
\end{tabular}




\begin{tabular}{cccc}
20 & 15 & 32 & 85 \\
$21-22$ & 20 & 33 & 90 \\
23 & 25 & 34 & 95 \\
$24-25$ & 30 & 35 & 96 \\
26 & 35 & 34 & 97 \\
27 & 40 & 35 & 98 \\
28 & 45 & 35 & 99 \\
28 & 50 & & \\
\hline
\end{tabular}

\section{DISCUSIÓN}

Este es el primer estudio que ha evaluado psicométricamente en Colombia la escala SWLS de satisfacción vital. En línea con la literatura publicada (Diener et al., 2013), la escala muestra adecuadas propiedades psicométricas en torno a la validez de constructo, así como a la consistencia y confiabilidad de la misma, lo que corresponde con múltiples estudios internacionales (Pavot \& Diener, 1993; Diener, \& Eunkook, 2003; Diener et al., 2013; Kowalska et al., 2017; Cwiek et al., 2017; Tsaousides et al., 2017; Yoder et al., 2017; Sarid et al 2017; Tessier et al., 2017; Lovereide \& Hagell, 2016; Mehamedagic et al., 2016), quienes hallaron una escala unidimensional, con un alfa de Cronbach alto. Los resultados muestran una apropiada consistencia interna entre los reactivos, correlaciones entre los elementos y el total de la prueba, y concordancia en la estructura factorial con la versión original, lo que sugiere una adecuada validez de constructo de la prueba, con apropiada confiabilidad y consistencia en la muestra bogotana de estudiantes universitarios. Estos resultados coinciden con lo informado en la revisión de Pavot y Diener (1993). Además, las correlaciones entre todos los reactivos de la escala en general, son similares con los datos informados en la revisión de Pavot y Diener (1993) y los resultados de este estudio muestran también que no hay diferencias en satisfacción en la vida entre hombres y mujeres, resultados que difieren con la investigación de Cárdenas et al. (2012) con estudiantes universitarios en Chile donde los puntajes favorecen a las mujeres y coinciden con los estudios de Pavot y Diener (1993) y Vázquez, et al., (2013) en los que no se encontraron diferencias entre sexos en estudios con población general. Además, es importante señalar la tendencia de respuesta de los universitarios que participaron en la investigación donde el $70 \%$ de ellos tienden a una respuesta positiva sobre la satisfacción con su vida, lo que coincide con estudios desarrollados en Colombia con el constructo felicidad (Cruz \& Torres, 2006; Sinisterra, 2007). 
Por otra parte, vale la pena mencionar un conjunto de limitaciones que presentó este estudio, donde las características de la muestra dificultan la posibilidad de generalizar los hallazgos al resto de la población, lo que sugiere desarrollar otros estudios con otras muestras poblacionales colombianas de diferentes estratos socioeconómicos, etnias y grupos etarios. Además, se sugiere realizar en próximos estudios análisis de invarianza factorial, validez convergente y evaluar la fiabilidad respecto a la estabilidad temporal; lo anterior, con el objetivo de comprobar los indicadores de validez y confiabilidad que requiere la escala y una mayor probabilidad de generalización y extrapolación de los resultados.

Finalmente, este estudio refiere que la Escala de Satisfacción con la vida (SWLS) es una escala válida y confiable, con baremaciones para universitarios bogotanos, por lo que se sugiere el uso de esta escala para futuros estudios que busquen análisis descriptivos acerca de la satisfacción con la vida en jóvenes colombianos a nivel general, comparaciones entre estratos, niveles educativos y demás variaciones que permitan dar cuenta de dicho constructo en población colombiana. Adicional, esta escala puede, con posteriores aplicaciones, convertirse como indicador práctico acerca de las condiciones emocionales de jóvenes, en tanto que servirá como un tamizaje de amplia aplicación en colegios y universidades acerca de la satisfacción con la vida, volviéndose un posible indicador de dificultades personales, por lo que se recomienda su utilización a nivel clínico y educativo.

Financiamiento: Artículo derivado de un trabajo conjunto entre el grupo de investigación Psychology \& Health de la Fundación Universitaria Sanitas, del Laboratorio de Medición y Evaluación de la Universidad de La Sabana, y del grupo Avances en Psicología Clínica y de la Salud de la Universidad de San Buenaventura, Bogotá.

Agradecimientos: A todos los participantes de la presente investigación.

\section{REFERENCIAS}

Alexandre, A., \& Guirardello, E. (2002). Adaptación cultural de instrumentos utilizados en salud ocupacional. Revista Panameña de Salud Pública, 11(2), 109-111. https://www.scielosp.org/article/rpsp/2002.v11n2/109-111/

Arango-Lasprilla, J.C., Moreno, A., Rogers, H., \& Francis, K. (2009). The effect of dementia patient's physical, cognitive, and emotional/behavioral problems on caregiver well-being: findings from a Spanish-speaking sample from Colombia, South America. American journal of Alzheimers disorders and other dementias, 24(5), 384-95. https://doi.org/10.1177/1533317509341465

Arango-Lasprilla, J., Plaza, S., Drew, A., Romero, J., Pizarro, J., Francis, K., \& Kreutzer, J. (2010). Family needs and psychosocial functioning of caregivers of individuals 
with spinal cord injury from Colombia, South America. NeuroRehabilitation, 27(1), 83-93. https://www.ncbi.nlm.nih.gov/pubmed/20634603. DOI: 10.3233 / NRE-2010-0583

Buliński, L., \& Błachnio, A. (2017). Health in old age, and patients' approaches to telemedicine in Poland. Ann Agric Environ Medical, 24(2), 322-328. https://www. ncbi.nlm.nih.gov/pubmed/28664717

Cárdenas, M., Barrientos, J., Bilbao, A., Páez, D., Gómez, F., \& Asun, D. (2012). Estructura factorial de la escala de satisfacción con la vida en una muestra de estudiantes universitarios chilenos. Revista Mexicana de Psicología, 29(2), 157-164. Recuperado de http://www.redalyc.org/pdf/2430/243030190006.pdf

Ćwiek, A., Czok, M., Kurczab, B., Kramarczyk, K., Drzyzga, K., \& Kucia, K. (2017). Association between depression and hemodialysis in-patient with chronic kidney disease. Psychiatria Danubina, 29(3), 499-503. Recuperado de http://www.hdbp. org/psychiatria_danubina/pdf/dnb_vol29_sup3/dnb_vol29_sup3_499.pdf

Cruz, J., \& Torres, J. (2006). "¿¿De qué depende la satisfacción subjetiva de los colombianos?". Cuadernos de Economía, 45, 131-154. Recuperado de http://www. scielo.org.co/scielo.php?script=sci_arttext\&pid=S0121-47722006000200007\&Ing=es\&nrm=iso\&tlng=es

Díaz, D., Stavraski, M., Blanco, A., \& Gandarillas, B. (2015). El componente eudaimónico de la satisfacción con la vida y el bienestar psicológico en culturas hispanas. Psicothema, 27(3), 247-253. http://www.psicothema.com/psicothema. asp?id=4260

Diener E., Emmons R., Larsen R. J., \& Griffin S. (1985). The Satisfaction with Life Scale. Journal of Personality Assessment, 49, 71-75. https://doi.org/10.1207/ s15327752jpa4901_13

Diener, E., \& Eunkook, M.S. (2003). National differences in subjective well-being. In D. Kahneman, E. Diener \& N. Schwartz (Eds.), Well-being: The foundations of hedonic psychology (pp. 434-450). New York: Russell Sage Foundation.

Diener, E. (1984). Subjective well-being. Psychological Bulletin, 95, 542-575. Recuperado de https://internal.psychology.illinois.edu/ ediener/Documents/ Diener_1984.pdf

Diener, E. (1994). Assessing subjective well -being progress and opportunities. Social Indicators Research, 31, 103-157. Recovered: https://www.montessoridenver. org/programs/student-assessment

Diener, E. (2000). Subjective well - being the science of happiness and a proposal for a national index. American Psychologist, 55(1), 34-43. http://dx.doi. org/10.1037/0003-066X.55.1.5

Diener, E., Tay, L., \& Oishi, S., (2013). Rising income and subjective Well-Being of Nations. Journal of Personality and social Psychology, 104(2), 267-276. https:// www.apa.org/pubs/journals/releases/psp-104-2-267.pdf

Diener, E., Wolsic, B. \& Fujita, F. (1995). Physical attractiveness and subjective well-being. Journal of Personality and Social Psychology, 69(1), 120-129. http:// dx.doi.org/10.1037/0022-3514.69.1.120

Gómez, V., Villegas, C., Barrera, F., \& Cruz, J. (2007). Factores predictivos de bienestar subjetivo en una muestra colombiana. Revista Latinoamericana de Psicología, 39(2), 311-325. Recuperado de http://www.redalyc.org/pdf/805/80539208.pdf

Gouveia, V., Milfont, T., Nunes, P., \& Peçanha de Miranda, J. (2009). Life satisfaction in Brazil: Testing the Psychometric Properties of the Satisfaction with Life Scale (SWLS) in Five Brazilian Samples. Social Indicators Research, 90, 267-277. https:// doi.org/10.1007/s11205-008-9257-0 
Goverman, J., Mathews, K., Nadler, D., Henderson, E., McMullen, K., Herndon, D., Meyer, W. 3rd, Fauerbach, J.A., Wiechman, S., Carrougher, G., Ryan, C.M., \& Schneider, J.C. (2016). Satisfaction with life after burn: A Burn Model System National Database Study. Burns. Journal of the international society for burn injuries, 42(5), 1067-1073. https://www.ncbi.nlm.nih.gov/pubmed/27215148

Jalali, L., Bigelow, P., McColl, S., Majowicz, S., Gohari, M., \& Waterhouse, R. (2016). Changes in quality of life and perceptions of general health before and after operation of wind turbines. Environmental Pollution, 216, 608-615. https://www. ncbi.nlm.nih.gov/pubmed/27321878

Kowalska, J., Gorackzo, A., Javorwka, L., \& Szczepańska, J. (2017). An Assessment of the Burden on Polish Caregivers of Patients with Dementia: A Preliminary Study. American Journal of Alzheimer's disease and Other Dementias, 32(8), 509-515. https://doi.org/10.1177/1533317517734350

López-Ortega, M., Torres-Castro, S., \& Rosas-Carrasco, O. (2016). Psychometric properties of the Satisfaction with Life Scale (SWLS): secondary analysis of the Mexican Health and Aging Study. Health Qual Life Outcomes, 14(1), 170. https:// doi.org/10.1186/s12955-016-0573-9

Lovereide, L., \& Hagell, P. (2016). Measuring life satisfaction in Parkinson disease and healthy controls using the satisfaction with life scale. PlosOne, 11(10). https://doi. org/10.1371/journal.pone.0163931

Mehamedagic, I., Santen, S., Jorgensen, S., \& Acosta, S. (2016). Life satisfaction in-patient with and without spinal cord ischemia after advanced endovascular therapy for extensive aortic disease at mid-term follow-up. Journal Rehabilitation Medicine, 48(10), 861-864. https://www.medicaljournals.se/jrm/content/ abstract/10.2340/16501977-2157

Montero, I., \& León, O. (2005). Sistema de clasificación del método en los informes de investigación en Psicología. International Journal of Clinical and Health Psychology, 5(1), 115-127. Recuperado de http://www.aepc.es/ijchp/articulos_ pdf/ijchp-136.pdf

Moyano, N., Martínez, M., \& Muñoz, M. (2013). Propiedades psicométricas de la escala de satisfacción con la vida de Diener. Revista Argentina de Clínica Psicológica, 22(1), 161-168. Recuperado de http://www.redalyc.org/articulo. oa?id=281931436008

Moreno, J.M., Nicholls, E., Ojeda, N., De los Reyes-Aragón, C.P., Rivera, D., \& Arango-Lasprilla, J.C. (2014). Caregiving in dementia and its impact on psychological functioning and health-related quality of life: Findings from Colombian sample. Journal of Cross Cultural Gerontology, 30(4), 393-408. https://www.ncbi. nlm.nih.gov/pubmed/26290365

Murillo-Muñoz, J., \& Molero-Alonso, F. (2016). Relación entre la satisfacción vital y otras variables psicosociales de migrantes colombianos en Estados Unidos. Revista Colombiana de Psicología, 25(1), 15-32. http://dx.doi.org/10.15446/rcp. v25n1.44308

Nader, M., Peñate, S., \& Sánchez, P. (2014). Predicción del bienestar y satisfacción en el trabajo: hacia un modelo de satisfacción saludable en Colombia. Estudios Gerenciales, 30(130), 31-39. https://doi.org/10.1016/j.estger.2014.02.006

Padros, F., Gutiérrez, C., \& Medina, M. (2015). Propiedades psicométricas de la escala de satisfacción con la vida (SWLS) de Diener en población de Michoacán (México). Avances en Psicología Latinoamericana, 33(2), 223-232. http://dx.doi. org/10.12804/apl33.02.2015.04

Paiva, B.S., de Camargos, M.G., Demarzo, M.M., Hervás, G., Vázquez, C., \& Paiva, C.E. (2016). The Pemberton Happiness Index: Validation of the Universal Portuguese 
version in a large Brazilian sample. Medicine (Baltimore), 95(38), e4915. https:// www.ncbi.nlm.nih.gov/pubmed/27661039

Pavot, W., \& Diener E. (1993). The affective and cognitive contest of self-reports measures of subjective well-being. Social Indicators Research, 28(1), 1-20. https:// doi.org/10.1007/BF01086714

Salazar, F. (2009). Felicidad en estudiantes de la facultad de salud de la Universidad Sur Colombiana. Revista Facultad de Salud, 1(2), 17-23. Recuperado de https:// www.journalusco.edu.co/index.php/rfs/article/view/42/59

Sarid, O., Slonim, V., Pereg, A., Friger, M., Sergienko, R., \& Schawarz. (2017). Coping strategies, satisfaction of life, and quality of life in Crohns disease: A gender perspective using structural equation modeling analysis. Plos One, 12(2). https:// doi.org/10.1371/journal.pone.0172779

Sinisterra, D. (2007). Colombian happiness: A look at life satisfaction and explanatory style. (Disertación Doctoral no publicada). Florida Atlantic University, Boca Raton, FL, EE.UU.

Sutter, M., Perrin, P.B., Chang, Y.P., Hoyos, G.R., Buraye, J.A. \& Arango-Lasprilla, J.C. (2014). Linking family dynamics and the mental health of Colombian dementia caregivers. American journal of Alzheimers disorders and other dementias, 29(1), 67-75. https://doi.org/10.1177/1533317513505128

Tessier, P., Blanchin, M., \& Sibille, V. (2017). Does the relationship between health-related quality of life and subjective well-being changeover time? An exploratory study among breast cancer patients. Social Science Medicine, 174, 96-103. https://www.ncbi.nlm.nih.gov/pubmed/28013110

Tsaousides, T., Spielman, L., Kajankova, M., Guetta, G., Gordon, W., \& Dams-O'Connor, K. (2017). Improving emotion regulation following web-based group intervention for individuals whit traumatic brain injury. Journal Head Trauma Rehabilitation, 32(5), 354-365. https://www.ncbi.nlm.nih.gov/pubmed/28891909

Vázquez, C., Duque, A., \& Hervás, G. (2013). Satisfaction with Life Scale in a Representative Sample of Spanish Adults: Validation and Normative Data. Spanish Journal of Psychology, 16(82), 1-15. https://www.ncbi.nlm.nih.gov/pubmed/24230945

Vera-Villarroel, P., Urzúa, M.A., Pavez, P., Celis-Atenas, K., \& Silva, J. (2012). Evaluation of Subjective Well-being: Analysis of the Satisfaction with Life Scale in Chilean Population. Universitas Psychologica, 11(3), 719-727. Recovered http://www. scielo.org.co/scielo.php?script=sci_arttext\&pid=S1657-92672012000300003

Yoder, L.H., McFall, D.C., \& Glaser, DN. (2017). Quality of life of burn survivors treated in the military burn center. Nursing Outlook, (17). http://isiarticles.com/bundles/ Article/pre/pdf/130662.pdf 